\title{
TRIGONOMETRIC PARALLAXES OF PLANETARY NEBULAE
}

\author{
H.C. HARRIS, C.C. DAHN, D.G. MONET AND J.R. PIER \\ U.S. Naval Observatory
}

PO Box 1149, Flagstaff, AZ 86002, USA

\begin{abstract}
The accuracy of parallaxes obtained with ground-based CCD cameras now often reaches or exceeds 0.5 milliarcseconds. This improved accuracy permits us to measure significant parallaxes and determine distances for the nearest planetary nebulae. At present, the central stars of 11 planetary nebulae have been observed as part of the USNO parallax program. We now have determined distances with accuracies better than 20 percent for four central stars and better than 50 percent for five more. This paper gives the present status of the program, a brief interpretation of the results, and future prospects.
\end{abstract}

\section{Introduction}

In observing stellar parallaxes, the use of cameras with CCD detectors offers three advantages over traditional photographic cameras. First, images have much higher signal/noise, limited by photon statistics rather than by grain noise. Second, the data are linear, allowing astrometry of both bright and faint stars with minimal systematic errors. Third, higher quantum efficiency allows the use of fainter reference stars which, in turn, allows a reference frame with smaller angular size less subject to distortion by Earth's atmosphere.

These advantages of astrometry with CCD cameras have resulted in a dramatic improvement in the accuracy of parallax measurements. Parallaxes can now be obtained with an accuracy of 0.5 milliarcseconds (mas) or better, based on data accurate to typically 3 mas in relative positions for a single observation (Monet et al. 1992 and in preparation). These results are almost an order of magnitude better than was typically achieved with photographic cameras during the 1970s and 1980s. 


\section{TRIGONOMETRIC PARALLAXES OF PN}

Until recently, planetary nebulae have eluded successful parallax measurements. Although the Yale Parallax Catalogue (van Altena et al. 1995) lists $24 \mathrm{PNe}$ with measured parallaxes, all but one (NGC 7293) have errors more than 8 mas, so these measurements obviously are not significant. The parallax of NGC 7293 was marginally detected in several studies, the best (according to the Yale Catalogue) with an error of 5 mas (Dahn et al. 1973; see Harrington \& Dahn 1980). Below we obtain $4.7 \pm 0.7$ mas, demonstrating the improved accuracy that has been achieved. Perhaps $23 \mathrm{PNe}$ or more have distances $\leq 400 \mathrm{pc}$ (Terzian 1993), so we might expect to make significant distance measurements (errors $\leq 20 \%$ ) for most of these PNe. We began this work in 1987 and gave a first report at the Innsbruck meeting (Pier et al. 1993). Here we present the current status of the program and plans for the future.

\section{Observations}

The USNO parallax program using a Texas Instruments 800x800 CCD was described by Monet et al. (1992). Observations of nine PNe were begun in 1987 using this camera. In 1992, observing began using a Tektronix $2048 \times 2048$ CCD. Four PNe (two in common with the TI800 program) are being observed with this camera. The much wider field of the Tek2048 CCD (11 arcminutes, compared with 3 arcminutes for the TI800 CCD), together with the greater full well of the Tek2048 pixels, allows selection of better reference stars (more stars, more distant stars, and stars with a better symmetrical distribution around the parallax star) that is important in sparse fields. Parallaxes determined with the TI800 CCD have a median error in the relative parallax of 1.0 mas, while parallaxes determined with the Tek2048 CCD at present have a median error of 0.7 mas, and the latter error is still dropping as more data are obtained. These results led us to terminate observing with the TI800 CCD in 1995. The current status of our PNe parallax data is shown in Table 1.

A correction for the finite distances of the reference stars must be applied to convert each observed relative parallax to absolute parallax. This correction is important for $\mathrm{PNe}$ with distances of several hundred pc, and becomes increasingly important as the error in the relative parallax drops. This correction is shown as $\Delta \pi$ in Table 1 . It has been determined both from spectroscopy and photometry of the reference stars. Spectra of many reference stars taken at Kitt Peak National Observatory (4-meter telescope, $7 \AA$ resolution) were used to measure strengths of 17 spectral features ( $\mathrm{H}$ lines, $\mathrm{Ca} \mathrm{K}, \mathrm{G}$ and $\mathrm{CN}$ bands, $\mathrm{MgH}, \mathrm{MgI}, \mathrm{Na} \mathrm{D}, \mathrm{CaI}$, and several Fe features). These indicies were used to determine the spectral type and luminosity class of each star and to check for any peculiarities (e.g. binary star, metal-poor 


\section{H.C. Harris}

star, emission lines, etc.). Second, BVI colors were measured for all stars; for red stars with $(\mathrm{V}-\mathrm{I})_{\mathrm{C}}>1.1$ they allow classification as a giant or dwarf.

The combined data give an estimate of the reddening and distance of each reference star. Any nearby stars are then rejected from the astrometric solutions. We have from 4 to 8 reference stars in the final solutions for the PNe observed with the TI800 camera, and from 7 to 13 stars for those observed with the Tek2048 camera. Note that for fields close to the galactic plane (particularly S216, and to a lesser extent NGC 6853), reddening is large enough to make the distance of each reference star more uncertain, adding to the error in $\Delta \pi$. For all fields, Table 1 shows that the reference stars are typically at distances of $1-2 \mathrm{kpc}$. At these distances, $\Delta \pi$ is fairly small and reasonably well-determined. Therefore, for each of these $\mathrm{PNe}$, the error in $\Delta \pi$ contributes only a small amount to the final error in the absolute parallax. Note that the use of faint reference stars is an important factor in keeping this contribution to the total error small. With faint reference stars, the error in the observed relative parallax dominates the total error.

TABLE 1. Observed Data

\begin{tabular}{|c|c|c|c|c|c|c|}
\hline \multirow[t]{2}{*}{ PN } & \multicolumn{2}{|c|}{ Number of } & \multirow[t]{2}{*}{ Epoch } & \multirow{2}{*}{$\begin{array}{c}\pi_{r e l} \\
(\mathrm{mas})\end{array}$} & \multirow{2}{*}{$\begin{array}{c}\Delta \pi \\
(\mathrm{mas})\end{array}$} & \multirow{2}{*}{$\begin{array}{c}\pi_{a b s} \\
\text { (mas) }\end{array}$} \\
\hline & Frames & Nights & & & & \\
\hline \multicolumn{7}{|c|}{ TI800 CCD Program: } \\
\hline NGC 6720 & 230 & 117 & $88.1-95.2$ & $0.58 \pm 0.45$ & $0.84 \pm 0.32$ & $1.42 \pm 0.55$ \\
\hline NGC 6853 & 146 & 68 & $88.4-95.6$ & $2.10 \pm 0.38$ & $0.53 \pm 0.20$ & $2.63 \pm 0.43$ \\
\hline A 21 & 66 & 53 & $87.9-95.8$ & $1.60 \pm 0.50$ & $0.25 \pm 0.10$ & $1.85 \pm 0.51$ \\
\hline A 24 & 18 & 18 & $88.0-95.2$ & $2.62 \pm 1.11$ & $0.49 \pm 0.17$ & $3.11 \pm 1.12$ \\
\hline A 29 & 26 & 25 & $89.2-95.2$ & $1.74 \pm 1.29$ & $0.43 \pm 0.19$ & $2.18 \pm 1.30$ \\
\hline A 31 & 22 & 18 & $88.2-93.1$ & $3.78 \pm 1.54$ & $0.97 \pm 0.48$ & $4.75 \pm 1.61$ \\
\hline A74 & 53 & 50 & $88.5-94.8$ & $0.96 \pm 0.62$ & $0.37 \pm 0.12$ & $1.33 \pm 0.63$ \\
\hline $\mathrm{S} 216$ & 103 & 40 & $89.7-94.9$ & $6.31 \pm 0.88$ & $1.06 \pm 0.47$ & $7.37 \pm 1.00$ \\
\hline PW1 & 61 & 31 & $87.9-95.8$ & $2.58 \pm 0.51$ & $0.54 \pm 0.24$ & $3.12 \pm 0.56$ \\
\hline \multicolumn{7}{|c|}{ TEK2048 CCD Program: } \\
\hline NGC 7293 & 123 & 87 & $92.6-96.6$ & $3.74 \pm 0.65$ & $0.96 \pm 0.36$ & $4.70 \pm 0.75$ \\
\hline S216 & 84 & 53 & $92.8-96.1$ & $7.06 \pm 0.50$ & $0.71 \pm 0.30$ & $7.77 \pm 0.58$ \\
\hline PW1 & 119 & 59 & $92.8-96.2$ & $1.07 \pm 0.47$ & $0.58 \pm 0.19$ & $1.65 \pm 0.51$ \\
\hline A7 & 35 & 31 & $93.9-96.2$ & $-0.51 \pm 0.59$ & $0.68 \pm 0.21$ & $0.17 \pm 0.63$ \\
\hline
\end{tabular}




\section{TRIGONOMETRIC PARALLAXES OF PN}

\section{Discussion}

The resulting "final" parallaxes are shown in Table 2. Obviously these results are not really final, because we are continuing to observe the four $\mathrm{PNe}$ on the Tek2048 program for at least one more season, and because we anticipate reobserving several (probably NGC 6853, A24, and A31) with the Tek2048 camera beginning soon. All these fields are likely to benefit from this additional observing. On the other hand, it appears that A7 is at a distance too large for us to be able to make a useful parallax measurement, and others (NGC 6720 and A74) are sufficiently distant that our data are only marginally useful.

Table 2 includes our determination of $\mathrm{E}(\mathrm{B}-\mathrm{V})$ for the central star from our observed $\mathrm{BVI}_{\mathrm{C}}$, assuming $(\mathrm{B}-\mathrm{V})_{0}=-0.38$ and $(\mathrm{V}-\mathrm{I})_{0}=-0.45$, and the derived $\mathrm{M}_{\mathrm{V}}$. The results are consistent with all stars having $\mathrm{M}_{\mathrm{V}} \sim 6.8$ except the central stars of NGC 6853 and A7, which appear to be brighter, and the central stars of A24, A29, and A31, which appear to be fainter. The latter are also the three $\mathrm{PNe}$ with the most uncertain distances, so their faint absolute magnitudes are only tentative.

TABLE 2. Results

\begin{tabular}{lccccll}
\hline \multicolumn{1}{c}{ PN } & $\begin{array}{c}\pi_{a b s} \\
(\mathrm{mas})\end{array}$ & $\begin{array}{c}\text { Distance } \\
(\mathrm{pc})\end{array}$ & $\mathrm{V}$ & $\mathrm{E}(\mathrm{B}-\mathrm{V})$ & $\mathrm{M}_{\mathrm{V}}(1 \sigma$ Range $)$ & Note \\
\hline NGC 6720 & $1.42 \pm 0.55$ & 704 & 15.75 & 0.08 & $6.26(5.20-6.97)$ & \\
NGC 6853 & $2.63 \pm 0.43$ & 380 & 14.05 & 0.09 & $5.87(5.48-6.19)$ & 1 \\
NGC 7293 & $4.70 \pm 0.75$ & 213 & 13.52 & 0.03 & $6.79(6.41-7.11)$ & 2 \\
A7 & $0.17 \pm 0.63$ & $\geq 700$ & 15.48 & 0.10 & $\leq 6.0$ & 2 \\
A21 & $1.85 \pm 0.51$ & 541 & 15.96 & 0.08 & $7.05(6.35-7.58)$ & \\
A24 & $3.11 \pm 1.12$ & $322:$ & 17.37 & 0.07 & $9.62(8.65-10.29)$ & 1,3 \\
A29 & $2.18 \pm 1.30$ & $459:$ & 18.31 & 0.14 & $9.57(7.60-10.59)$ & 3 \\
A31 & $4.75 \pm 1.61$ & $211:$ & 15.52 & 0.07 & $8.69(7.79-9.32)$ & 1,3 \\
A74 & $1.33 \pm 0.63$ & 752 & 17.05 & 0.12 & $7.29(5.90-8.14)$ & \\
S216 & $7.67 \pm 0.50$ & 130 & 12.62 & 0.08 & $6.79(6.65-6.93)$ & \\
PW1 & $2.31 \pm 0.38$ & 433 & 15.53 & 0.14 & $6.91(6.52-7.24)$ & 2 \\
\hline
\end{tabular}

1. Reobserve with Tek2048 CCD.

2. Will improve with more data.

3. Insufficient data, preliminary result.

In Table 3, a comparison is made between the distances determined here and those from several other recent discussions of PNe distances. In general, the agreement is about what might be expected given the known uncertainties of $\mathrm{PNe}$ distances. We find somewhat larger distances for A7 


\section{H.C. Harris}

and PW1 than have been determined in other studies, and a somewhat smaller distance for A24. Note that A24, A29, and A31 are preliminary results based on fewer data than is desirable. For the remaining objects, the distances determined here tend to be on the large end of the range found in previous studies. For example, we have observed eight of the 11 stars listed by Terzian (1993, his Table 3 ) as having distances < 300 pc. We find only three of these eight stars to be within $300 \mathrm{pc}$.

TABLE 3. Comparison with Other Studies

\begin{tabular}{lcccccc}
\hline \multirow{2}{*}{ PN } & $\begin{array}{c}\text { Distance in pc: } \\
\text { This }\end{array}$ & CKS $^{1}$ & Terzian & VZ $^{2}$ & NS $^{3}$ & Pottasch \\
& Paper & 1992 & 1993 & 1994 & 1995 & 1996 \\
\hline NGC 6720 & 704 & 872 & $\geq 500$ & 1000 & 990 & 500 \\
NGC 6853 & 380 & 262 & 270 & 400 & 550 & 360 \\
NGC 7293 & 213 & 157 & 160 & 400 & $\ldots$ & 280 \\
A7 & $\geq 700$ & 216 & 220 & $\ldots$ & $\ldots$ & 550 \\
A21 & 541 & $\ldots$ & 270 & $\ldots$ & 600 & 500 \\
A24 & $322:$ & 525 & $\geq 500$ & $\ldots$ & $\ldots$ & 600 \\
A29 & $459:$ & $\ldots$ & 410 & $\ldots$ & $\ldots$ & $\ldots$ \\
A31 & $211:$ & 233 & 240 & $\ldots$ & $\ldots$ & 400 \\
A74 & 752 & $\ldots$ & 230 & $\ldots$ & 750 & 850 \\
S216 & 130 & $\ldots$ & 40 & $\ldots$ & 110 & 130 \\
PW1 & 433 & 141 & 240 & $\ldots$ & 470 & 300 \\
\hline${ }^{1}$ Cahn et al. 1992 & & & & & \\
${ }^{2}$ Van de Steene \& Zijlstra 1994 & & & & \\
${ }^{3}$ Napiwotzki \& Schönberner 1995 & & & &
\end{tabular}

\section{Future Improvements}

Beside the parallax program at the Naval Observatory, Anguita, GutierrezMoreno, and Moreno are observing A29, YM 29, and He2-11 (Anguita 1996, private communication) and expect to get results in 1997. The Hipparcos Input Catalogue (Turon et al. 1992) includes the central stars of 14 PNe. Most of these have distance estimates from the literature of $\sim 1 \mathrm{kpc}$, and of course most have magnitudes near the faint limit for Hipparcos. Therefore, unfortunately, probably only three or four $\mathrm{PNe}$ will have significant parallax measurements made by Hipparcos.

Several other PNe are likely to be at distances where useful parallax measurements can be made now from the ground. As well as repeating a couple of the PNe in Table 2, we hope to add NGC 3242, DHW5, HW6, 


\section{TRIGONOMETRIC PARALLAXES OF PN}

Ton 320, PG0108+101, and RE1738+665 to the Tek2048 program soon. In addition, we hope to observe A35, LT5, and BD+28 4211 with a new camera including a $5^{m}$ neutral density filter (necessary for these bright central stars), beginning next year. We hope other researchers will include some of these objects in their studies to compare distances determined with other methods. If other $\mathrm{PNe}$ are of particular importance and are likely to be at a distance closer than $500 \mathrm{pc}$, please contact us to request their addition to the program.

At present, we believe systematic errors in the absolute parallaxes are still smaller than the random observational errors. If so, the errors should continue to improve to $\leq 0.5$ mas for all objects as sufficient data accumulate. Judging by present results and estimated distances for new objects, it is possible that we will have $10 \mathrm{PNe}$ or more with parallaxes giving distances determined to better than $20 \%$ by the time of the next meeting in this series. More importantly, the expanded sample will give a more definite indication of the systematic distance scale and space density for nearby PNe.

\section{References}

Cahn, J.H., Kaler, J.B., \& Stanghellini, L. 1992, A\&AS, 94, 399

Dahn, C.C., Behall, A.L., \& Christy, J.W. 1973, PASP, 85, 224

Harrington, R.S., \& Dahn, C.C. 1980, AJ, 85, 454

Monet, D.G., Dahn, C.C., Vrba, F.J., Harris, H.C., Pier, J.R., Luginbuhl, C.B., \& Ables, H.D. 1992, AJ, 103, 638

Napiwotzki, R., \& Schönberner, D. 1995, A\&A, 301, 545

Pier, J.R., Harris, H.C., Dahn, C.C., \& Monet, D.G. 1993, in Proc. IAU Symp. No. 155, Planetary Nebulae, ed. R. Weinberger \& A. Acker (Kluwer, Dordrecht), p. 175

Pottasch, S.R. 1996, A\&A, 307, 561

Terzian, Y. 1993, in Proc. IAU Symp. No. 155, Planetary Nebulae, ed. R. Weinberger \& A. Acker (Kluwer, Dordrecht), p. 109

Turon, C., et al. 1992, The Hipparcos Input Catalogue. European Space Agency Publication.

van Altena, W.F., Lee, J.T., and Hoffleit, E.D. 1995, The General Catalogue of Trigonometric Stellar Parallaxes, 4th Edition. (Yale University Observatory, New Haven).

Van de Steene, G.C., \& Zijlstra, A.A. 1994, A\&AS, 108, 485 